\title{
Amalgamation, Absoluteness, and Categoricity
}

\author{
John T. Baldwin* \\ University of Illinois at Chicago
}

December 27, 2009

\begin{abstract}
We describe progress on determining the categoricity spectrum of infinitary sentences. We expound the connections with first order logic and specify several set theoretic problems which arise. We provide a simple example of the failure of amalgamation for a complete sentence of $L_{\omega_{1}, \omega}$ and we sketch some folklore results concerning the absoluteness of basic model theoretic notions in infinitary logic.
\end{abstract}

\section{The Universe is Wide or Deep}

Shelah made the following rough conjecture: Let $\boldsymbol{K}$ be a reasonable class of models.

Either for some $\lambda$, there are many models of cardinality $\lambda$ or there are models of arbitrarily large cardinality.

Our metaphor requires some explanation. 'The universe' should perhaps be 'each universe'; universe refers to all models in a specific class. It turns out that this question depends very much on the choice of 'reasonable'. It also seems to be sensitive to the choice of axioms of set theory. In order to give a precise formulation of the conjecture we have to specify 'many' and the notion of a 'reasonable class'. In general 'many' should mean $2^{\lambda}$; but in important cases that have been proved, it is slightly smaller.

As is often the case there are some simplifying assumptions in this area that have been internalized by specialists but obscure the issues for other logicians. We try to explain a few of these simplifications and sketch some of the major results.

Some historical background will help clarify the issues. Much model theoretic research in the 60's focussed on general properties of first order and infinitary logic. A number of results seemed to depend heavily on extensions of ZFC. For example, both Keisler's proof that two structures are elementarily equivalent if and only if they have isomorphic ultrapowers and Chang's proof of two cardinal transfer required GCH. In general, even the existence of saturated models depends on the GCH. Shelah removed

\footnotetext{
${ }^{*}$ This article is a synthesis of the paper given in Singapore with later talks in 2009, including the MittagLeffler Institute and reflects discussions with set theorists during my stay there. Baldwin was partially supported by NSF- 0500841 .
} 
the set-theoretic hypothesis from Keisler's theorem. But various versions of two cardinal transfer were proven to require $\mathrm{GCH}$ and even large cardinal hypotheses. See [CK73].

The invention of stability theory radically recast the subject of model theory. E.g., for various classes in the stability hierarchy, it is straightforward to characterize in ZFC exactly in which cardinals there are saturated models. And for the best behaved theories the answers is: all cardinals. Further, for countable stable theories Shelah and Lachlan independently showed that two cardinal transfer between any pair of cardinalities is true in ZFC. Moreover, the fundamental notions of first order stability theory are absolute.

For first order logic, our guiding question is trivial ${ }^{1}$. If a theory has an infinite model then it has arbitrarily large models. The question is interesting for theories in logics which fail the upward Löwenheim-Skolem theorem. The notion of an Abstract Elementary Class (AEC) provides a general framework for analyzing such classes. But as we show in the next section the conjecture is trivially false in that case. It is not too difficult to find in ZFC examples (Example 2.1) of AEC that have no model above $\aleph_{1}$ but that are $\aleph_{1}$-categorical [She09a, Bal09]. And in $L_{\omega_{1}, \omega}(Q)$, it is consistent (via Martin's axiom) that are $\aleph_{1}$-categorical sentences with no model of cardinality greater than $2^{\aleph_{0}}$. But those sentences have many models in $2^{\aleph_{0}}$. In this note we describe how for $L_{\omega_{1}, \omega}$, there are major advances on the target problem. They use extensions of ZFC but rather mild ones; the initials below refer to the 'Weak Continuum Hypothesis' and the 'Very Weak Continuum Hypothesis':

WGCH: Cardinal exponentiation is an increasing function.

VWGCH: Cardinal exponentiation is an increasing function below $\aleph_{\omega}$.

This leaves us with two more precise questions.

1. Does the proof of the conjecture for $L_{\omega_{1}, \omega}$ (see Section 4) really need VWGCH?

2. Is the conjecture 'eventually true' for AEC' $\mathrm{s}^{2}$

Much of core mathematics studies either properties of particular structures of size at most the continuum or makes assertions that are totally cardinal independent. E.g., if every element of a group has order two then the group is abelian. Model theory and even more clearly infinitary model theory allows the investigation of 'structural properties' that are cardinal dependent such as: existence of models, spectra of stability, and number of models and existence of decompositions. Often these properties can be tied to global conditions such as the existence of a 'good' notion of dependence.

\section{Abstract Elementary Classes}

We begin by discussing the notion of an abstract elementary class. The examples show that this is too broad a class to be 'reasonable' for our target problem. But some positive

\footnotetext{
${ }^{1}$ The main gap theorem, every first order theory either eventually has the maximal number of models or the number of models is bounded by a small function, has the same flavor. And in fact the argument for this result arose after Shelah's consideration of the infinitary problems.

${ }^{2}$ For much positive work in this direction see [She09a].
} 
results can be proved in this general setting; this generality exposes more clearly what is needed for the argument by avoiding dependence on accidental syntactic features.

An abstract elementary class $(\boldsymbol{K}, \prec \boldsymbol{K})$ is a collection of structures for a fixed vocabulary $\tau$ that satisfy, where $A \prec \boldsymbol{K} B$ means in particular $A$ is a substructure of $B$,

1. If $A, B, C \in \boldsymbol{K}, A \prec_{\boldsymbol{K}} C, B \prec_{\boldsymbol{K}} C$ and $A \subseteq B$ then $A \prec_{\boldsymbol{K}} B$;

2. Closure under direct limits of $\prec \boldsymbol{K}^{\text {-embeddings; }}$

3. Downward Löwenheim-Skolem. If $A \subset B$ and $B \in \boldsymbol{K}$ there is an $A^{\prime}$ with $A \subseteq A^{\prime} \prec_{\boldsymbol{K}} B$ and $|A|^{\prime} \leq|A|=\mathrm{LS}(\boldsymbol{K})$.

The invariant $\operatorname{LS}(\boldsymbol{K})$, is a crucial property of the class. The class of well-orderings satisfies the other axioms (under end extension) but is not an AEC.

Two easy examples are: First order and $L_{\omega_{1}, \omega}$-classes; $L(Q)$ classes have Löwenheim-Skolem number $\aleph_{1}$. For the second case one has to be careful about the definition of $\prec \boldsymbol{K}$ - being an $L(Q)$-elementary submodel does not work ( a union of a chain can make $(Q x) \phi(x)$ become true even if it is false along the chain).

The notion of AEC has been reinterpreted in terms of category theory by Kirby: "Abstract Elementary Categories" [Kir08] and by Lieberman: "AECs as accessible categories" [Lie].

It is easy to see that just AEC is too weak a condition for the general conjecture.

Example 2.1 The set $\boldsymbol{K}=\left\{\alpha: \alpha \leq \aleph_{1}\right\}$ with $\prec \boldsymbol{K}$ as initial segment is an AEC with $\aleph_{1}$ countable models. It is $\aleph_{1}$-categorical and satisfies both amalgamation and joint embedding but is not $\omega$-Galois stable [Kue08]. And in fact there is no model of $\aleph_{2}$. So this universe is neither wide nor deep.

Let's clarify the specific meaning of the amalgamation property in this context.

Definition 2.2 The class $\boldsymbol{K}$ satisfies the amalgamation property if for any situation with $A, M, N \in \boldsymbol{K}$ :

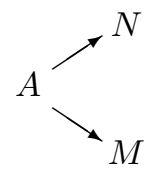

there exists an $N_{1}$ such that

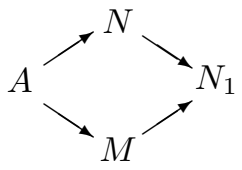


Note that we have required the base structure $A$ to be in $\boldsymbol{K}$; this is sometimes referred to as 'model amalgamation'. Requiring amalgamation over arbitrary substructures $A$ is a much stronger condition, which fails for important natural examples such as Zilber's pseudo-exponential field [Zil04]. There is much work in homogenous model theory where the stronger homogeneity condition is assumed.

The existence of amalgamations is an absolutely fundamental problem for AEC and for any study of infinitary logic. In first order logic it is easy to show that for complete theories amalgamation always holds over models with $\prec$ as elementary extension. And it holds over arbitrary subsets of models if $T$ admits elimination of quantifiers. Here is a basic example of failure for a complete sentence of $L_{\omega_{1}, \omega}$.

Example 2.3 Let $T$ be the first order theory in a language with binary relation symbols $\left\langle E_{i}: i<\omega\right\rangle$ that asserts the $E_{i}$ are infinitely many refining equivalence relations with binary splitting.

Using $L_{\omega_{1}, \omega}$ the equivalence relation $E_{\infty}$, the intersection of the given equivalence realations, is definable. Add two unary predicates (blue and red) and the infinitary axioms

1. Each $E_{\infty}$-class contains infinitely many elements.

2. Every element of an $E_{\infty}$-class is red or every element is blue.

3. Blue and red divide the $E_{\infty}$-classes into dense and codense sets.

Now it is easy to check that these axioms are $\aleph_{0}$-categorical but fail amalgamation (since a new path may be either red or blue).

We introduced the notion of abstract elementary class in this paper in order to state One Completely General Result [She83b, Bal09].

Theorem 2.4 [WGCH] Suppose $\lambda \geq \operatorname{LS}(\boldsymbol{K})$ and $\boldsymbol{K}$ is $\lambda$-categorical. If amalgamation fails in $\lambda$ there are $2^{\lambda^{+}}$models in $\boldsymbol{K}$ of cardinality $\kappa=\lambda^{+}$.

The argument uses weak diamond and is primarily combinatorial; it proceeds directly from the definition of an AEC. The result fails under $M A+\neg C H$. The same example is presented in both [She09a, Bal09]. It is an AEC (even given by a theory in $L(Q)$ ) which fails amalgamation in $\aleph_{0}$, but becomes $\aleph_{1}$-categorical in a forcing extension. But it remains open whether there are such examples in $L_{\omega_{1}, \omega}$.

\section{From $L_{\omega_{1}, \omega}$ to first order}

We begin by translating the problem from infinitary logic into the study of specific subclasses of models of first order theories. This removes the distraction of developing new notions of each syntactic idea (e.g. type) for every each fragment of $L_{\omega_{1}, \omega}$. More subtly, for technical reasons we need to restrict to complete sentences in $L_{\omega_{1}, \omega}$. (This restriction to complete sentences is automatic in the first order case but its legitimacy is only proved in certain cases for infinitary logic). 
Definition 3.1 For $\Delta$ a fragment of $L_{\omega_{1}, \omega}, a \Delta$-theory $T$ is $\Delta$-complete if for every $\Delta$-sentence $\phi, T \models \phi$ or $T \models \neg \phi$. We may write complete when $\Delta=L_{\omega_{1}, \omega}$.

Definition 3.2 A model $M$ of a first order theory is called atomic if each finite sequence from $M$ realizes a principal type over the empty set-one generated by a single formula.

A set $A \subset M$ is atomic if the analogous condition holds: each finite sequence from A realizes a principal type over the empty set-generated by a single formula.

The study of categoricity (at least from $\aleph_{1}$ upwards), in $L_{\omega_{1}, \omega}$ can be translated to the study of atomic models of a first order theory. This is non-trivial. The argument begins with a fundamental result from the early 60 's.

Theorem 3.3 (Chang/Lopez-Escobar) Let $\psi$ be a sentence in $L_{\omega_{1}, \omega}$ in a countable vocabulary $\tau$. Then there is a countable vocabulary $\tau^{\prime}$ extending $\tau$, a first order $\tau^{\prime}$ theory $T$, and a countable collection of $\tau^{\prime}$-types $\Gamma$ such that reduct is a 1-1 map from the models of $T$ which omit $\Gamma$ onto the models of $\psi$.

The proof is straightforward. E.g., for any formula $\psi$ of the form $\bigwedge_{i<\omega} \phi_{i}$, add to the language a new predicate symbol $R_{\psi}(\mathbf{x})$. Add to $T$ the axioms

$$
(\forall \mathbf{x}) R_{\psi}(\mathbf{x}) \rightarrow \phi_{i}(\mathbf{x})
$$

for $i<\omega$ and omit the type $p=\left\{\neg R_{\psi}(\mathbf{x})\right\} \cup\left\{\phi_{i}: i<\omega\right\}$.

Thus we have restricted to the models of a theory that omit a family $\Gamma$ of types, but it may realize some non-principal types. Shelah observed that if $T$ had only countably many types then applying the same argument again gives a $T^{\prime}$ in an expanded language such that the required interpretation is obtained by omitting all non-principal types. That is, the object of study is the atomic models of $T^{\prime}$. This further reduction is technically important. In particular it implies $\omega$-categoricity.

But why can we assume that the $T$ associated with $\phi$ has only countably many types over the empty set? We need a few definitions to give an explanation.

Definition 3.4 Fix a sentence $\phi \in \Delta_{\omega_{1}, \omega}$ and let $\Delta$ be a countable fragment of $L_{\omega_{1}, \omega}$ containing $\phi$.

1. A $\tau$-structure $M$ is $\Delta$-small if $M$ realizes only countably many $\Delta$-types (over the empty set).

2. An $L_{\omega_{1}, \omega}$-sentence $\phi$ is $\Delta$-small if there is a set $X$ countable of complete $\Delta$-types over the empty set and each model realizes some subset of $X$.

'small' means $\Delta=L_{\omega_{1}, \omega}$

It is easy to see that if $M$ is small then $M$ satisfies a complete sentence. If $\phi$ is small then Scott's argument for countable models generalizes and there is a complete sentence $\psi_{\phi}$ such that: $\phi \wedge \psi_{\phi}$ has a countable model. So $\psi_{\phi}$ implies $\phi$. But $\psi_{\phi}$ is not in general unique. For example $\phi$ might be just the axioms for algebraically closed fields. 
Two choices for $\psi_{\phi}$ are the Scott sentence of the prime field and the Scott sentence for the model of transcendence degree $\aleph_{0}$. Only the second has an uncountable model.

We can make an appropriate choice of $\psi_{\phi}$ if $\phi$ is $\aleph_{1}$-categorical. There are two ingredients in the choice.

Theorem 3.5 (Shelah) If $\phi$ has an uncountable model $M$ that is $\Delta$-small for every countable $\Delta$ and $\phi$ is $\kappa$-categorical then $\phi$ is implied by a complete sentence $\psi$ with a model of cardinality $\kappa$.

This result appears first in [She83a]. It is retold in [Bal09]; in [Bal07], we adapt the argument to give a model theoretic proof of a result of Makkai (obtained by admissible set theory) that a counterexample to Vaught's conjecture is not $\aleph_{1}$-categorical. The crux of Shelah's argument is an appeal to the non-definability of well-order in $L_{\omega_{1}, \omega}$.

The second step is to require that for each countable fragment $\Delta$ there are only countably many $\Delta$-types over the empty set. If $\phi$ has arbitrarily large models this is easy by using Ehrenfeucht-Mostowski models. But if not, the only known argument is from few models in $\aleph_{1}$ and depends on a subtle argument of Keisler [Kei71] (See also Appendix C of [Ba109].)

Theorem 3.6 (Keisler) If $\phi$ has $<2^{\aleph_{1}}$ models of cardinality $\aleph_{1}$, then each model of $\phi$ is $\Delta$-small for every countable $\Delta$.

Now Theorem's 3.5 and 3.6 immediately yield.

Theorem 3.7 (Shelah) If $\phi$ has $<2^{\aleph_{1}}$ models of cardinality $\aleph_{1}$, then there is a complete sentence $\psi$ such that $\psi$ implies $\phi$ and $\psi$ has an uncountable model. In particular, if $\phi$ is $\aleph_{1}$-categorical there is a Scott sentence for the model in $\aleph_{1}$.

But we have the following question, which is open if $\kappa>\aleph_{1}$.

Question 3.8 If $\phi$ is a $\kappa$-categorical must there be a Scott sentence for the model in $\kappa$.

Thus for technical work we will consider the class of atomic models of first order theories. Our notion of type will be the usual first order one - but we will want to focus on a subset of the Stone space.

Definition 3.9 Let $A$ be an atomic set; $S_{\text {at }}(A)$ is the collection of $p \in S(A)$ such that if $\boldsymbol{a} \in \mathbb{M}$ realizes $p, A \boldsymbol{a}$ is atomic.

Here $\mathbb{M}$ is the monster model for the ambient theory $T$; in interesting cases it is not atomic. And the existence of a monster model for the atomic class is a major project (It follows from excellence.).

Definition 3.10 $\boldsymbol{K}$ is $\lambda$-stable if for every model $M$ in $\boldsymbol{K}$ (thus necessarily atomic) with cardinality $\lambda,\left|S_{a t}(M)\right|=\lambda$.

The insistence that $M$ be a model is essential. The interesting examples of pseudoexponential field, covers of Abelian varieties and the basic examples of Marcus and Julia Knight all are $\omega$-stable but have countable sets $A$ with $\left|S_{\text {at }}(A)\right|>\aleph_{0}$.

With somewhat more difficulty than the first order case, one obtains: 
Theorem 3.11 For a class $\boldsymbol{K}$ of atomic models, $\omega$-stable implies stable in $\kappa$ for all $\kappa$.

A fundamental result in model theory is Morley's proof that an $\aleph_{1}$-categorical first order theory is $\omega$-stable. This argument depends on the compactness theorem in a number of ways. The key idea is to construct an Ehrenfeucht-Mostowski model over a well-order of cardinality $\aleph_{1}$. Such a model realizes only countably many types over any countable submodel. But the existence of the model depends a compactness argument in the proof of the Ehrenfeucht-Mostowski theorem. Further, this only contradicts $\omega$ stability because amalgamation allows the construction from a model $M_{0}$ in $\aleph_{0}$ that has uncountably many types over it an elementary extension $M_{1}$ of $M_{0}$ with power $\aleph_{1}$ that realizes all of them. And again amalgamation in the first order case is a consequence of compactness. In $L_{\omega_{1}, \omega}$, the work of Keisler and Shelah evades the use of compactness - but at the cost of set theoretic hypotheses.

Theorem 3.12 (Keisler-Shelah) Let $\boldsymbol{K}$ be the atomic models of a countable first order theory. If $\boldsymbol{K}$ is $\aleph_{1}$-categorical and $2^{\aleph_{0}}<2^{\aleph_{1}}$ then $\boldsymbol{K}$ is $\omega$-stable.

This proof uses WCH directly and weak diamond via 'The Only Completely General Result'. That is, from amalgamation failure of $\omega$-stability yields a model of cardinality $\aleph_{1}$ that realizes uncountably many types from $S_{\text {at }}(M)$ for a countable model $M$. Naming the elements of $M$ yields a theory which has uncountably many types over the empty set. Thus by Theorem 3.6 the new theory has $2^{\aleph_{1}}$ models in $\aleph_{1}$ and (since $2^{\aleph_{0}}<2^{\aleph_{1}}$ ) so does the original theory.

Is $\mathrm{CH}$ is necessary? More precisely, does $\mathrm{MA}+\neg \mathrm{CH}$ imply there is a sentence of $L_{\omega_{1}, \omega}$ that is $\aleph_{1}$-categorical but

a) is not $\omega$-stable

b) does not satisfy amalgamation even for countable models.

There is such an example in $L_{\omega_{1}, \omega}(Q)$ but Laskowski (unpublished) showed the example proposed for $L_{\omega_{1}, \omega}$ by Shelah[She87, She09a] fails. The previous question is a specific strategy for answering the next question.

Question 3.13 Is categoricity in $\aleph_{1}$ of a sentence of $L_{\omega_{1}, \omega}$ absolute (with respect suitable forcings)?

By suitable, I mean that, e.g., it is natural to demand cardinal preserving. This result has resisted a number of attempts although as we lay out in Section 5, many other fundamental notions of the model theory of $L_{\omega_{1}, \omega}$ are absolute.

\section{The Conjecture for $L_{\omega_{1}, \omega}$}

Using the notion of splitting, a nice theory of independence can be defined for $\omega$-stable atomic classes [She83a, She83b, Bal09]. This allows the formulation of the crucial notion of excellence and the proof of a version of Morley's theorem. We won't discuss the details but sketch the most important application. These results are non-trivial but the exposition of the entire situation in [Bal09] occupies less than 100 pages. 
Definition 4.1 Let $\boldsymbol{K}$ be the class of models of a sentence of $L_{\omega_{1}, \omega} . K$ is excellent if $\boldsymbol{K}$ is $\omega$-stable and any of the following equivalent conditions hold.

For any finite independent system of countable models with union $C$ :

1. $S_{a t}(C)$ is countable.

2. There is a unique primary model over $C$.

3. The isolated types are dense in $S_{a t}(C)$.

See [Bal09] for details of the notation. A primary model is a particulary strong way of choosing a prime model over $C$. Note excellence is a condition on countable models. It has the following consequence for models in all cardinalities.

Theorem 4.2 ( Shelah (ZFC)) If an atomic class $\boldsymbol{K}$ is excellent and has an uncountable model then

1. K has models of arbitrarily large cardinality;

2. Categoricity in one uncountable power implies categoricity in all uncountable powers.

This result is in ZFC but extensions of set theory are used to obtain excellence. Recall that by VWGCH we mean the assertion: $2^{\aleph_{n}}<2^{\aleph_{n+1}}$ for $n<\omega$.

Theorem 4.3 ( Shelah (VWGCH)) An atomic class $\boldsymbol{K}$ that has at least one uncountable model and is categorical in $\aleph_{n}$ for each $n<\omega$ is excellent.

The requirement of categoricity below $\aleph_{\omega}$ is essential. Baldwin-Kolesnikov [BK09] (refining [HS90]) show:

Theorem 4.4 For each $2 \leq k<\omega$ there is an $L_{\omega_{1}, \omega}$-sentence $\phi_{k}$ such that:

1. $\phi_{k}$ has an atomic model in every cardinal.

2. $\phi_{k}$ is categorical in $\mu$ if $\mu \leq \aleph_{k-2}$;

3. $\phi_{k}$ is not categorical in any $\mu$ with $\mu>\aleph_{k-2}$;

4. $\phi_{k}$ has the (disjoint) amalgamation property;

There is one further refinement on the 'wide' vrs 'deep' metaphor. How wide?

Definition 4.5 We say

1. $\boldsymbol{K}$ has few models in power $\lambda$ if $I(\boldsymbol{K}, \lambda)<2^{\lambda}$.

2. $\boldsymbol{K}$ has very few models in power $\aleph_{n}$ if $I\left(\boldsymbol{K}, \aleph_{n}\right) \leq 2^{\aleph_{n-1}}$.

These are equivalent under GCH. And Shelah argues on the last couple of pages of [She83b] (see also [She0x]) that they are equivalent under $\neg O^{+}$. But in general we have a theorem and a conjecture[She83a, She83b]. 
Theorem 4.6 (Shelah) (For $n<\omega, 2^{\aleph_{n}}<2^{\aleph_{n+1}}$.) An atomic class $\boldsymbol{K}$ that has at least one uncountable model and that has very few models in $\aleph_{n}$ for each $n<\omega$ is excellent.

Conjecture 4.7 (Shelah) (For $n<\omega, 2^{\aleph_{n}}<2^{\aleph_{n+1}}$.) An atomic class $\boldsymbol{K}$ that has at least one uncountable model and that has few models in $\aleph_{n}$ for each $n<\omega$ is excellent.

\section{Absoluteness}

As remarked in the introduction, one of the significant attributes of first order stability theory is that the basic notions: stable, $\omega$-stable, superstable, $\aleph_{1}$-categoricity can be seen absolute in the strongest way. We sketch proofs of similar results, except the open $\aleph_{1}$-categoricity, for $L_{\omega_{1}, \omega}$. The results in this section are folklore; we are indebted for discussions with Alf Dolich, Paul Larson, Chris Laskowski, and Dave Marker for clarifying the issues. One of the few places these issues have been addressed in print is [She09b].

For example a first order theory $T$ is unstable just if there is a formula $\phi(\mathbf{x}, \mathbf{y})$ such for every $n$

$$
T \models\left(\exists \mathbf{x}_{1}, \ldots \mathbf{x}_{n} \exists \mathbf{y}_{1}, \ldots \mathbf{y}_{n}\right) \bigwedge_{i<j} \phi\left(\mathbf{x}_{i}, \mathbf{y}_{j}\right) \wedge \bigwedge_{i \geq j} \neg \phi\left(\mathbf{x}_{i}, \mathbf{y}_{j}\right)
$$

This is an arithmetic statement and so is absolute by basic properties of absoluteness [Kun80, Jec87]. Similarly, the existence of a countable structure that satisfies a condition (such as being a model or being atomic) given by a conjunction of first order properties is $\Sigma_{1}^{1}$-property.

In first order logic, $\omega$-stability is $\Pi_{1}^{1}$; there is no consistent $\operatorname{tree}^{3}\left\{\phi_{i}^{\sigma(i)}\left(x_{\sigma}, \boldsymbol{a}_{\sigma}\right.\right.$ । $\left.n): \sigma \in 2^{\omega}, i<\omega\right\}$. For the class of atomic models the definition is slightly more complicated. And we need the full strength of the Shoenfield absoluteness lemma.

\section{Theorem 5.1 (Shoenfield absoluteness Lemma) If}

1. $V \subset V^{\prime}$ are models of $Z F$ with the same ordinals and

2. $\phi$ is a lightface $\Pi_{2}^{1}$ predicate of a set of natural numbers

then for any $A \subset N, V \models \phi(A)$ iff $V^{\prime} \models \phi(A)$.

Note that this trivially gives the same absoluteness criteria for $\Sigma_{2}^{1}$-predicates.

Lemma 5.2 Let $T$ be a countable first order theory. The property that a class of atomic models of $T$ is $\omega$-stable is given by a $\Sigma_{2}^{1}$ formula of set theory and so the property is absolute.

Proof. The class of atomic models of $T$ is $\omega$-stable if and only if

1. the isolated types are dense in $S(\emptyset)$;

\footnotetext{
${ }^{3}$ We use the convention that $\phi^{\sigma(i)} \phi(x)$ denotes $\phi(x)$ or $\neg \phi(x)$ depending on whether $\sigma(i)$ is 0 or 1 .
} 
2. there is a countable atomic model $M$ of $T$ and there is a list $\left\langle p_{n}: n \in \omega\right\rangle$ of the (first order) types over $M$ such that

(*) for any $\bar{b}$ realizing $p_{i}$ and any $\bar{a} \in M, \operatorname{tp}(\bar{a}, \bar{b})$ is isolated in $S(T)$

and for any $p \in S(M)$ satisfying $(*)$ there is an $\mathrm{n}$ such that $p_{n}=p$

The second version has the form $(\exists M)\left(\exists p_{n}\right)(\forall p)$. This is a $\Sigma_{2}^{1}$ formula of set theory.

Similarly we show excellence is a $\Pi_{2}^{1}$-property. Independent families of models [She83b, Bal09] are indexed by subsets of $n$ with strictly less than $n$ elements; we denote this partial order by $\mathcal{P}^{-}(n)$. We list the crucial definitions for excellence.

Definition 5.3 1. A complete type $p$ over $A$ splits over $B \subset A$ if there are $\mathbf{b}, \mathbf{c} \in A$ which realize the same type over $B$ and a formula $\phi(\mathbf{x}, \mathbf{y})$ with $\phi(\mathbf{x}, \mathbf{b}) \in p$ and $\neg \phi(\mathbf{x}, \mathbf{c}) \in p$.

2. Let $A B C$ be atomic. We write $A \downarrow B$ and say $A$ is free or independent from $B$ $C$ over $C$ if for any finite sequence $\boldsymbol{a}$ from $A, \operatorname{tp}(\boldsymbol{a} / B)$ does not split over some finite subset of $C$.

Lemma 5.4 Let $T$ be a countable first order theory. The class of atomic models of $T$ is excellent if and only if for any finite set of models $\left\{A_{s}: s \in \mathcal{P}^{-}(n)\right\}$ that form an independent system, there is a countable atomic model $M$ of $T$ that is primary over $C=\bigcup\left\{A_{s}: s \in \mathcal{P}^{-}(n)\right\}$.

Proof. Here we have universal quantifiers over finite sequences of models (using a pairing function, this is quantifying over a single real) and then an existential quantifier for the required additional model. The stipulation that the diagram is independent requires repeated use of the statement $A \underset{C}{\downarrow} B$, where $A, B, C$ are finite unions of the models in the independent system. This requires quantification over finite sequences from the $M_{i}$; thus, it is arithmetic. The assertion that $M$ is primary over $C$ is a second existential quantifier enumerating elements of $M$.

The following remark is implicit in [GS86].

Lemma 5.5 The property that a complete sentence of $L_{\omega_{1}, \omega}$ has arbitrarily large models is absolute.

Proof. A complete sentence in $L_{\omega_{1}, \omega}$ has arbitrarily large models if and only if the associated countable Skolem theory $T$ has an Ehrenfeucht-Mostowski model over a countable set of order indiscernibles, which omits each of a countable (from the completeness of $\phi$ ) set of types $\Gamma$. We can rewrite this as:

There exists a countable sequence $C$ of constants, that are indiscernible and for each finite sequence $\mathbf{c}$ from $C$, each Skolem term $\tau$ of appropriate arity, and each type $p \in \Gamma$ there is a formula $\phi(\mathbf{x}) \in p$ with $\neg \phi(\tau(\mathbf{c}))$. This is a $\Sigma_{1}^{1}$ formula, which proves the result.

Finally, following Lessmann [Les05, Bal09], we prove that the absolute 'BaldwinLachlan'-characterization of first order $\aleph_{1}$-categoricity has a natural translation to the 
$L_{\omega_{1}, \omega}$ situation; this translation is absolute and in ZFC it implies $\aleph_{1}$-categoricity. But we do not see how to derive it from $\aleph_{1}$-categoricity without using the Continuum hypothesis. We need some definitions. To be a bit more specific we speak of Vaughtian triples instead of Vaughtian pairs.

Definition 5.6 The formula $\phi(x, \mathbf{c})$ with $\mathbf{c} \in M \in \boldsymbol{K}$, is big if for any $M^{\prime} \supseteq A$ with $M^{\prime} \in \boldsymbol{K}$ there exists an $N^{\prime}$ with $M^{\prime} \prec_{\boldsymbol{K}} N^{\prime}$ and with a realization of $\phi(x, \mathbf{c})$ in $N^{\prime}-M^{\prime}$.

This definition has no requirements on the cardinality of $M, M^{\prime}, N^{\prime}$ so it is saying that $\phi(\mathbf{x}, \mathbf{c})$ has as many solutions as the size of the largest models in $\boldsymbol{K}$. This condition is equivalent to one on countable models. A translation of Lemma 25.2 of [Bal09] gives:

Lemma 5.7 Let $A \subseteq M$ and $\phi(x, \mathbf{c})$ be over $A$. The following are equivalent.

1. There is an $N$ with $M \prec N$ and $c \in N-M$ satisfying $\phi(x, \mathbf{c})$;

2. $\phi(x, \mathbf{c})$ is big.

The significance of this remark is that it makes ' $\phi(x, \mathbf{c})$ is big' a $\Sigma_{1}^{1}$ predicate.

Definition 5.8 1 . A triple $(M, N, \phi)$ where $M \prec N \in \boldsymbol{K}$ with $M \neq N$, $\phi$ defined over $M, \phi$ big, and $\phi(M)=\phi(N)$ is called a Vaughtian triple.

2. We say $\boldsymbol{K}$ admits $(\kappa, \lambda)$, witnessed by $\phi$, if there is a model $N \in \boldsymbol{K}$ with $|N|=\kappa$ and $|\phi(N)|=\lambda$ and $\phi$ is big.

Now we have the partial characterization.

Lemma 5.9 Let $\boldsymbol{K}$ be a class of atomic models. If $\boldsymbol{K}$ is $\omega$-stable and has no Vaughtian triples then $\boldsymbol{K}$ is $\aleph_{1}$-categorical. The hypothesis of this statement is $\Sigma_{2}^{1}$.

Proof. The sufficiciency of the condition is found by tracing results in [Bal09]. $\omega$-stability gives the existence of a quasiminimal formula $\phi$. Note from the proof of Theorem 24.1 that $\omega$-stability is sufficient to show that there are prime models over independent subsets of cardinality $\aleph_{1}$. (The point of excellence is that higher dimensional amalgamation is needed to extend this result to larger sets.) So if $|M|=\aleph_{1}$, there is an $N \prec{ }_{K} M$ which is prime over a basis for $\phi(M)$. As noted in Chapter 2 of [Bal09], this determines $N$ up to isomorphism (again without heavy use of excellence because we are in $\aleph_{1}$ ). So we are done unless $N \underset{\precsim}{\precsim} M$. But then Löwenheim-Skolem gives us a countable Vaughtian triple, contrary to hypothesis.

Consequence 5.10 Let $\psi$ be a complete sentence of $L_{\omega_{1}, \omega}$ and $\boldsymbol{K}$ the associated class of atomic models. Suppose either:

1. $\boldsymbol{K}$ has arbitrarily large members and $\boldsymbol{K}$ has amalgamation in $\aleph_{0}$, or

2. $2^{\aleph_{0}}<2^{\aleph_{1}}$. 
Then $\aleph_{1}$-categoricity of $\psi$ is absolute.

Proof. Each hypothesis implies the characterization in Lemma 5.9. $\quad \square_{5.10}$

Note, the hypothesis of condition 1 ) is absolute. It seems unlikely that $\aleph_{1}$ categoricity implies the existence of arbitrarily large models in $\boldsymbol{K}$; but no counterexample has yet been constructed. The use of the continuum hypothesis is central to proving that $\aleph_{1}$-categoricity implies amalgamation and $\omega$-stability. But [FK0x] have shown (employing standard forcings) that for each AEC $\boldsymbol{K}$ that fails amalgamation in $\aleph_{0}$, there is a model of set theory such that in that model $2^{\aleph_{0}}=2^{\aleph_{1}}, \boldsymbol{K}$ continues to fail amalgamation in $\aleph_{0}$, and $\boldsymbol{K}$ has $2^{\aleph_{1}}$ models in $\aleph_{1}$.

\section{Conclusion}

The spectrum problem for first order theories motivated many technical developments that eventually had significant algebraic consequences. A similar possibility for infinitary logics is suggested by Zilber's program [Zil06, Zil04]. But the basic development is far more difficult and less advanced. The notion of excellence provides one useful context. And others are being developed under the guise of abstract elementary classes and metric abstract elementary classes. But while first order stability theory is developed in ZFC, the current development of the model theory of $L_{\omega_{1}, \omega}$ uses a (rather weak) extension of set theory: the VWGCH. This raises both model theoretic and set theoretic questions. The proof of the 'one completely general result' Theorem 2.4 is a fundamentally combinatorial argument using no sophisticated model theoretic lemmas. The current proof uses $2^{\lambda}<2^{\lambda^{+}}$. Can this hypothesis be removed?

Like first order logic such fundamental definitions of $L_{\omega_{1}, \omega}$ as satisfaction, $\omega$ stablity, and excellence are absolute. But while $\aleph_{1}$-categoricity is seen (by a model theoretic argument) to be absolute in the first order case, this issue remains open for $L_{\omega_{1}, \omega}$.

\section{References}

[Bal07] J.T. Baldwin. Vaught's conjecture, do uncountable models count? Notre Dame Journal of Formal Logic, pages 1-14, 2007.

[Bal09] John T. Baldwin. Categoricity. Number 51 in University Lecture Notes. American Mathematical Society, 2009. www.math.uic.edu/ ${ }^{\sim}$ jbaldwin.

[BK09] John T. Baldwin and Alexei Kolesnikov. Categoricity, amalgamation, and tameness. Israel Journal of Mathematics, 170, 2009. also at www . math. uic.edu $\backslash \sim \backslash j$ baldwin.

[CK73] C.C. Chang and H.J Keisler. Model theory. North-Holland, 1973. 3rd edition 1990.

[FK0x] Sy-David Friedman and Martin Koerwien. On absoluteness of categoircity in aecs. preprint, 200x. 
[GS86] R. Grossberg and Saharon Shelah. On the number of non isomorphic models of an infinitary theory which has the order property part A. Journal of Symbolic Logic, 51:302-322, 1986.

[HS90] Bradd Hart and Saharon Shelah. Categoricity over $P$ for first order $T$ or categoricity for $\phi \in 1_{\omega_{1} \omega}$ can stop at $\aleph_{k}$ while holding for $\aleph_{0}, \cdots, \aleph_{k-1}$. Israel Journal of Mathematics, 70:219-235, 1990.

[Jec87] T. Jech. Multiple Forcing, volume 88 of Cambridge Topics in Mathematics. Cambridge University Press, 1987.

[Kei71] H.J Keisler. Model theory for Infinitary Logic. North-Holland, 1971.

[Kir08] Jonathan Kirby. Abstract elementary categories. http://people. maths.ox.ac.uk/ kirby/pdf/aecats.pdf, 2008.

[Kue08] D. W. Kueker. Abstract elementary classes and infinitary logics. Annals of Pure and Applied Logic, pages 274-286, 2008.

[Kun80] K. Kunen. Set Theory, An Introduction to Independence Proofs. North Holland, 1980.

[Les05] Olivier Lessmann. An introduction to excellent classes. In Yi Zhang, editor, Logic and its Applications, Contemporary Mathematics, pages 231-261. American Mathematical Society, 2005.

[Lie] M. Lieberman. Accessible categories vrs aecs. preprint:www math. Isa. umich.edu/ liebermm/vita.html.

[She83a] S. Shelah. Classification theory for nonelementary classes. I. the number of uncountable models of $\psi \in L_{\omega_{1} \omega}$ part A. Israel Journal of Mathematics, 46:3:212-240, 1983. paper 87a.

[She83b] S. Shelah. Classification theory for nonelementary classes. I. the number of uncountable models of $\psi \in L_{\omega_{1} \omega}$ part B. Israel Journal of Mathematics, 46;3:241-271, 1983. paper 87b.

[She87] Saharon Shelah. Classification of nonelementary classes II, abstract elementary classes. In J.T. Baldwin, editor, Classification theory (Chicago, IL, 1985), pages 419-497. Springer, Berlin, 1987. paper 88: Proceedings of the USA-Israel Conference on Classification Theory, Chicago, December 1985; volume 1292 of Lecture Notes in Mathematics.

[She09a] S. Shelah. Classification Theory for Abstract Elementary Classes. Studies in Logic. College Publications iwww.collegepublications.co.uk $i$, 2009. Binds together papers 88r, 300, 600, 705, 734, 838 with introduction E53.

[She09b] S. Shelah. Model theory without choice? categoricity. Journal of Symbolic Logic, 74:361-401, 2009. 
[She0x] S. Shelah. Non-structure in $\lambda^{++}$using instances of WGCH. paper 838, 200x.

[Zi104] B.I. Zilber. Pseudo-exponentiation on algebraically closed fields of characteristic 0. Annals of Pure and Applied Logic, 132:67-95, 2004.

[Zil06] B.I. Zilber. Covers of the multiplicative group of an algebraically closed field of characteristic 0. J. London Math. Soc., pages 41-58, 2006. 